\title{
Características de los pacientes con cardiopatías congénitas complejas atendidos en un hospital de concentración
}

\section{Characteristics of patients with complex congenital heart disease treated in a concentration hospital}

\author{
Arturo García-Galicia, Gustavo A. Flores-Gonzaga, David E. Herrera-Solano, Guillermo Ordóñez-Toquero, \\ Roberto Morales-Moreno y Álvaro J. Montiel-Jarquín*
}

Unidad Médica de Alta Especialidad, Hospital de Especialidades, Centro Médico Nacional "General de División Manuel Ávila Camacho", Instituto Mexicano del Seguro Social, Puebla, México

\begin{abstract}
Resumen
Introducción: Las cardiopatías congénitas, el segundo tipo de malformación en niños, se presentan en 6-8 casos de cada 1,000 recién nacidos vivos. Son alteraciones estructurales cardiovasculares localizadas en el situs, ventrículos o grandes vasos y son detectadas en la etapa neonatal o lactante. Objetivo: Determinar las características clínicas de los pacientes con cardiopatías complejas, atendidos en el Hospital de Especialidades de Puebla del Instituto Mexicano del Seguro Social. Método: Estudio descriptivo, transversal y retrospectivo en pacientes menores de 15 años, con cardiopatías complejas durante 2017 y 2019. Se registró edad, género, lugar de origen, cuadro clínico, exposición a teratógenos, edad materna y tipo de cardiopatía. Se utilizó estadística descriptiva y $\chi^{2}$. Resultados: 47 pacientes, $51.1 \%$ niñas. Con signos más frecuentes, soplo (93.6\%) y cianosis (42.6\%). Edad materna media de 28 años. Exposición a teratógenos (8.5\%). Diagnósticos: Coartación aórtica (46.8\%), tetralogía de Fallot (14.9\%) y anomalía de Ebstein (10.6\%). Sin significado estadístico en cuanto a diagnóstico, origen, ni factor de riesgo. Conclusiones: Predomina el sexo femenino, edad media al diagnóstico 21.6 meses; los signos más frecuentes son el soplo y la cianosis.
\end{abstract}

Palabras clave: Cardiopatías complejas. Cardiopatías congénitas. Cianosis. Soplos cardiacos.

\begin{abstract}
Background: Congenital heart diseases, the second type of malformation in children, occur in 6-8 per 1000 live newborns. They are structural cardiovascular alterations at situs, ventricles or great vessels and detected in neonatal or infant stages. Objective: To determine the clinical characteristics of patients with complex heart disease treated at Hospital de Especialidades de Puebla from Instituto Mexicano del Seguro Social. Method: A descriptive, cross-sectional and retrospective study was carried out in children under 15 years old, with complex heart disease, treated during 2017 and 2019. The following variables were taken: age, gender, origin, clinical manifestations, teratogen exposure, maternal age, type of heart disease. Descriptive statistics and $\chi^{2}$ were used. Results: 47 cases were recruited, 51.1\% female. Most frequent signs: murmur (93.6\%) and cyanosis (42.6\%). Medium maternal age of 28 years old. Teratogens exposure: $8.5 \%$. Diagnoses: Aortic coarctation (46.8\%), Fallot's tetralogy (14.9\%) and Ebstein's anomaly (10.6\%). There was no statistical significance about diagnostic, origin and risk factor. Conclusion: Female sex predominates, mean age at diagnosis 21.6 months; the most frequently signs were murmur and cyanosis.
\end{abstract}

Key words: Complex heart disease. Congenital heart disease. Cyanosis. Heart murmurs.

Correspondencia:

*Álvaro J. Montiel-Jarquín

E-mail: dralmoja@ hotmail.com

1405-9622/@ 2020 Sociedad Médico-Quirúrgica del Hospital Juárez de México, A.C. Publicado por Permanyer. Este es un artículo open access bajo la licencia CC BY-NC-ND (http://creativecommons.org/licenses/by-nc-nd/4.0/).
Fecha de recepción: 01-10-2020

Fecha de aceptación: 05-11-2020

DOI: 10.24875/RHJM.20000115
Disponible en internet: 05-05-2021 Rev Hosp Jua Mex. 2021;88(2):76-80

www.revistahospitaljuarez.com 


\section{Introducción}

Las cardiopatías congénitas se presentan en 6-8 de cada 1,000 recién nacidos vivos; hacen referencia a las alteraciones estructurales cardiovasculares a nivel del situs, los ventrículos o los grandes vasos, con lo que pueden alterarse las conexiones/relaciones de dichas estructuras. Se detectan mayormente en la etapa neonatal o lactante y son la segunda causa de malformación en la edad pediátrica, siguiendo a las malformaciones del sistema nervioso central. Su detección oportuna repercute en el pronóstico $^{1,2}$.

Las cardiopatías congénitas complejas representan el $25 \%$ de las cardiopatías. El $30 \%$ de los casos se muestran clínicamente sanos en los primeros días de vida $^{1,2}$. Un factor importante en el diagnóstico y para mejorar la planeación es el diagnóstico mediante ecocardiografía fetal, que aporta datos sobre defectos estructurales a nivel cardiovascular en la etapa prenatal; además, ya se han implementado avances para otorgar tratamiento en esta etapa del desarrollo, como el intervencionismo fetal o la valvuloplastia aórtica o pulmonar. Existen diversos métodos para el diagnóstico de las cardiopatías, tales como la ecocardiografía tridimensional, tomografía computarizada, resonancia magnética y Doppler ${ }^{3}$. El tratamiento inicialmente es médico, sin embargo, se requerirá en muchas ocasiones de procedimientos invasivos como la cirugía o el cateterismo intervencionista ${ }^{4}$.

Las cardiopatías congénitas se encuentran dentro de las causas principales de muerte infantil, por lo que exigen un conjunto de especialistas y científicos para mejorar la atención y el pronóstico, y también recopilar las experiencias en el seguimiento clínico ${ }^{5}$.

\section{Objetivo}

Determinar las características clínicas de los pacientes con cardiopatías complejas, atendidos en la Unidad Médica de Alta Especialidad del Hospital de Especialidades de Puebla del Centro Médico Nacional "General de División Manuel Ávila Camacho", del Instituto Mexicano del Seguro Social (IMSS) en Puebla, México, en el periodo 2017-2019.

\section{Material y métodos}

El presente estudio fue aprobado por el Comité Local de Investigación y Ética en Investigación en Salud 2101, con número de registro R-2018-2101-058.
Es un estudio transversal, descriptivo, en pacientes atendidos en la Unidad Médica de Alta Especialidad del Hospital de Especialidades de Puebla del Centro Médico Nacional "General de División Manuel Ávila Camacho", del IMSS, en el periodo de 2017 a 2019. El servicio de cardiopediatría de este hospital recibea pacientes derechohabientes del IMSS provenientes de los estados de Puebla, Tlaxcala y Oaxaca, en México. Se seleccionaron expedientes de pacientes menores de 15 años de edad con cardiopatía compleja, atendidos en el servicio de cardiopediatría en el periodo 2017-2019, no se excluyó a ningún paciente. Se eliminaron los expedientes clínicos con información incompleta. Se tomaron las siguientes variables: edad, sexo, lugar de origen, cuadro clínico, exposición a teratógenos, edad materna, cromosomopatía y tipo de cardiopatía. Para el análisis estadístico se utilizó estadística descriptiva y $\chi^{2}$ con el programa estadístico SPSS para Mac de IBM v.23.

\section{Resultados}

El total de pacientes atendidos en el Hospital fue de 47 , no hubo pacientes eliminados. Se encontró un predominio del género femenino, en relación con el lugar de origen lugar de los pacientes, principalmente el área metropolitana de la ciudad de Puebla, el área de Tehuacán y del estado de Tlaxcala como los más representativos. Con respecto a la edad de diagnóstico, la media fue de 28.1 con mínimo de 1 mes y un máximo de 96 meses. La edad materna media fue 28.1 años, mínimo 17 y máximo 44. El detalle se muestra en la tabla 1.

En relación con el tipo de cardiopatía, los diagnósticos más frecuentes fueron: coartación aórtica, seguido de tetralogía de Fallot y anomalía de Ebstein (Fig. 1). Los signos más frecuentemente descritos fueron el soplo cardiaco, con $93.6 \%$ ( $n=44$ ), y la cianosis con $42.6 \%(n=20)$. Entre los factores de riesgo: la exposición a teratógenos en el $8.5 \%(n=4)$, en madres empleadas de maquiladoras; el $91.5 \%(n=43)$ no tuvo ninguna exposición (Tabla 2).

La cardiopatía compleja más frecuente fue la coartación de la aorta, y con lugar de origen mayoritariamente la Ciudad de Puebla, seguida de la tetralogía de Fallot (49.2\% de casos en la zona de Tehuacán), observando para el resto de las patologías una distribución más dispersa según el lugar de origen. Esto arrojó un resultado por $\chi^{2}=59.3, p=0.356$ (Tabla 3 ). 
Tabla 1. Características demográficas y edades de los pacientes con diagnóstico de cardiopatías congénitas complejas del servicio de cardiopediatría del Centro Médico Nacional Manuel Ávila Camacho

\begin{tabular}{|c|c|c|}
\hline $\begin{array}{l}\text { Edad al diagnóstico (meses) } \\
\text { Media } \\
\text { Mínimo } \\
\text { Máximo }\end{array}$ & \multicolumn{2}{|c|}{$\begin{array}{c}21.6 \\
1 \\
96\end{array}$} \\
\hline $\begin{array}{l}\text { Edad materna (años) } \\
\text { Media } \\
\text { Mínima } \\
\text { Máximo }\end{array}$ & \multicolumn{2}{|c|}{$\begin{array}{c}28.1 \\
17 \\
44\end{array}$} \\
\hline & $\begin{array}{l}\text { Frecuencia } \\
\qquad(n=47)\end{array}$ & $\begin{array}{c}\text { Porcentaje } \\
(\%)\end{array}$ \\
\hline $\begin{array}{l}\text { Sexo } \\
\text { Femenino } \\
\text { Masculino }\end{array}$ & $\begin{array}{l}23 \\
24\end{array}$ & $\begin{array}{l}48.9 \\
51.1\end{array}$ \\
\hline $\begin{array}{l}\text { Lugar de origen } \\
\text { Cholula } \\
\text { Huachinango } \\
\text { Huejotzingo } \\
\text { Cd. de Puebla } \\
\text { Tehuacán } \\
\text { Zacapoaxtla }\end{array}$ & $\begin{array}{c}2 \\
1 \\
1 \\
32 \\
5 \\
1\end{array}$ & $\begin{array}{c}4.3 \\
2.1 \\
2.1 \\
68.1 \\
10.6 \\
2.1\end{array}$ \\
\hline $\begin{array}{l}\text { Total por Estado } \\
\text { Puebla } \\
\text { Oaxaca } \\
\text { Tlaxcala }\end{array}$ & $\begin{array}{c}42 \\
1 \\
4\end{array}$ & $\begin{array}{c}89.3 \\
2.1 \\
8.5\end{array}$ \\
\hline
\end{tabular}

\section{Discusión}

De acuerdo con la literatura internacional, se estima que hasta el $85 \%$ de los niños recién nacidos que tienen cardiopatías congénitas podrá alcanzar la edad adulta dependiendo de la detección, oportunidad y el acceso al tratamiento de manera precoz. La mayoría de los pacientes que llegan a sobrevivir presentan lesiones residuales 0 secuelas a otros niveles ${ }^{5}$.

En la serie de casos que se presenta, el promedio de edad al diagnóstico es de 21.6 meses. Ello destaca, ya que está bien establecido que a menor edad de diagnóstico, el pronóstico suele ser mejor ${ }^{5}$. Esto concuerda con otros reportes latinoamericanos, en donde la mayoría de los pacientes se diagnostican antes de los 24 meses $^{6}$.

Los signos clínicos más frecuentemente encontrados (soplo cardiaco y cianosis) son los signos que usualmente se reportan en pacientes con estas patologías ${ }^{7}$.

En México, según el Instituto Nacional de Estadística y Geografía, aproximadamente en la década del 2001 al 2010 fallecieron 31,991 niñas y niños antes de cumplir un año de vida, y 68 de cada 1,000 nacidos vivos fallecieron por afecciones cardiacas ${ }^{8}$. El pronóstico de vida de los niños con cardiopatía compleja dependerá de diferentes factores, como la complejidad, la edad al diagnóstico, el tipo y el momento del tratamiento otorgado ${ }^{9}$. En México, las que son tratadas quirúrgicamente, son los defectos del septum ventricular, tetralogía de Fallot, persistencia del conducto arterioso, conexión anómala total de venas pulmonares y coartación de aorta, con una mortalidad global del 7.5\% comparada con los países desarrollados, que es del $4 \%{ }^{10}$.

A diferencia de otros estudios, en el nuestro la coartación aórtica, la tetralogía de Fallot y la anomalía de Ebstein fueron las cardiopatías más frecuentes. Al parecer estos resultados tienden a cambiar según el lugar donde se realice el estudio ${ }^{11}$. La tetralogía de Fallot es la cardiopatía congénita cianógena más frecuente y es una de las más investigadas. La gran prevalencia de casos con tetralogía de Fallot provenientes de Tehuacán representa una ventana de oportunidad para la investigación, de la misma forma que el porcentaje elevado de cardiopatías provenientes del área metropolitana de la ciudad de Puebla, aunque esto podría explicarse porque el hospital se encuentra en esta área, siendo esta la mancha urbana de mayor concentración poblacional en la región. Asimismo, los diversos procesos ambientales son un factor de riesgo importante, ya que cambia la estructura a nivel celular y molecular. Entre estos factores, que han podido ser identificados desde 1940 hasta nuestra actualidad, algunos han actuado sobre la embriogénesis cardiaca, y cumpliendo otras premisas de acción teratogénica, también enfermedades como las maternas infecciosas y no infecciosas, factores químicos, y sustancias químicas ambientales como humo de cocina, monóxido de carbono y tabaquismo ${ }^{12,13}$.

En nuestra población se observa que existe una relación con la exposición a teratógenos, que es del $8.5 \%$ de todos los casos, también hay exposición a solventes industriales, las maquiladoras son las principales, siendo esta también una de las principales fuentes de trabajo de Puebla y Tehuacán, además de que del $100 \%$ de casos de tetralogía de Fallot, el $28.3 \%$ presentaron la misma exposición; de la misma manera con los pacientes que tienen coartación aórtica, donde el $9.1 \%$ presentó el mismo antecedente. Se preconiza la importancia de actuar sobre los factores etiológicos y desencadenantes en la etapa preconcepcional, aunque el $90 \%$ de estos tiene una etiología desconocida y se han presentado enfermos los hijos de madres sin factores de riesgo, por lo que 


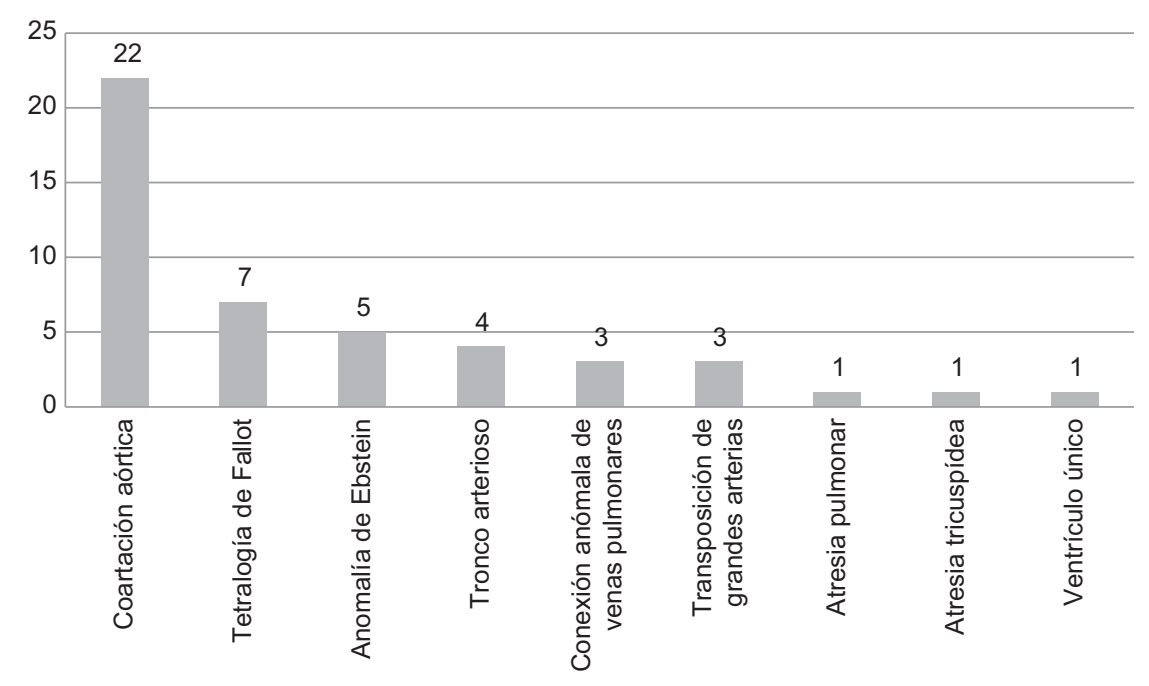

Figura 1. Diagnósticos de las cardiopatías complejas del servicio de cardiopediatría del Centro Médico Nacional Manuel Ávila Camacho.

Tabla 2. Asociación diagnóstica de los pacientes con cardiopatías complejas y la exposición a teratógenos

\begin{tabular}{|l|c|c|c|c|c|c|c|c|c|}
\hline & $\begin{array}{c}\text { Trasposición } \\
\text { de GA }\end{array}$ & $\begin{array}{c}\text { Atresia } \\
\text { tricuspídea }\end{array}$ & $\begin{array}{c}\text { Tronco } \\
\text { arterioso }\end{array}$ & $\begin{array}{c}\text { Tetralogía } \\
\text { de Fallot }\end{array}$ & $\begin{array}{c}\text { Conexión } \\
\text { anómala de } \\
\text { VP }\end{array}$ & $\begin{array}{c}\text { Atresia } \\
\text { pulmonar }\end{array}$ & $\begin{array}{c}\text { Coartación } \\
\text { aórtica }\end{array}$ & $\begin{array}{c}\text { Anomalía } \\
\text { de } \\
\text { Ebstein }\end{array}$ & $\begin{array}{c}\text { Ventrículo } \\
\text { único }\end{array}$ \\
\hline Total $(n=47)$ & 3 & 1 & 4 & 7 & 3 & 1 & 22 & 5 & 1 \\
\hline $\begin{array}{l}\text { Exposición a teratógenos } \\
\text { No } \\
\text { Sí }\end{array}$ & 3 & 1 & 4 & 5 & 3 & 1 & 20 & 5 & 1 \\
\hline
\end{tabular}

VP: venas pulmonares; GA: grandes arterias.

el ecocardiograma fetal deviene en una Financiamiento

prioridad ${ }^{14-16}$.

El tamaño de la muestra limita mucho el estudio, sin embargo, es la casuística atendida en un hospital de concentración de $3{ }^{\text {er }}$ nivel de atención. Aún así es interesante ver la distribución del tipo de cardiopatía según las distintas áreas geográficas de esta región del país.

Podemos concluir que en los pacientes con cardiopatías congénitas complejas atendidos en Puebla predomina el sexo femenino, con edad media al diagnóstico de 21.6 meses, siendo la cianosis y el soplo los principales datos clínicos. Se debe enfatizar en hacer una buena semiótica ante la sospecha de una cardiopatía congénita y registrar adecuadamente los signos vitales, para así tener un mejor control y seguimiento.
La presente investigación no ha recibido ninguna beca específica de agencias de los sectores público, comercial o sin ánimo de lucro.

\section{Conflicto de intereses}

Los autores declaran no tener conflicto de intereses alguno.

\section{Responsabilidades éticas}

Protección de personas y animales. Los autores declaran que para esta investigación no se han realizado experimentos en seres humanos ni en animales. 
Tabla 3. Asociación entre diagnóstico y lugar de origen en pacientes con cardiopatías complejas

\begin{tabular}{|c|c|c|c|c|c|c|c|c|}
\hline Diagnóstico & $\begin{array}{l}\text { Total } \\
(\mathrm{n}=47)\end{array}$ & $\begin{array}{l}\text { Cd. de } \\
\text { Puebla* }\end{array}$ & Tehuacán* & Cholula* & Huauchinango* & Zacapoaxtla* & Tlaxcala & Oaxaca \\
\hline Coartación aórtica & 22 & $\begin{array}{c}17 \\
77.3 \%\end{array}$ & $\begin{array}{c}1 \\
4.5 \%\end{array}$ & & & & $\begin{array}{c}3 \\
13.6 \%\end{array}$ & $\begin{array}{c}1 \\
4.5 \%\end{array}$ \\
\hline Tetralogía de Fallot & 7 & $\begin{array}{c}2 \\
66.7 \%\end{array}$ & $\begin{array}{c}3 \\
4.5 \%\end{array}$ & & & $\begin{array}{c}1 \\
14.3 \%\end{array}$ & $\begin{array}{c}1 \\
14.3 \%\end{array}$ & \\
\hline Anomalía de Ebstein & 5 & $\begin{array}{c}3 \\
60 \%\end{array}$ & & $\begin{array}{c}1 \\
20 \%\end{array}$ & $\begin{array}{c}1 \\
20 \%\end{array}$ & & & \\
\hline Tronco arterioso & 4 & $\begin{array}{c}3 \\
75 \%\end{array}$ & & & & & $\begin{array}{c}1 \\
25 \%\end{array}$ & \\
\hline Conexión anómala de VP & 3 & $\begin{array}{c}2 \\
66 \%\end{array}$ & & $\begin{array}{c}1 \\
33 \%\end{array}$ & & & & \\
\hline Trasposición de GA & 3 & $\begin{array}{c}3 \\
100 \%\end{array}$ & & & & & & \\
\hline Atresia pulmonar & 3 & $\begin{array}{c}2 \\
66 \%\end{array}$ & $\begin{array}{c}1 \\
33 \%\end{array}$ & & & & & \\
\hline Atresia cuspídea & 1 & $\begin{array}{c}1 \\
100 \%\end{array}$ & & & & & & \\
\hline Ventrículo único & 1 & $\begin{array}{c}1 \\
100 \%\end{array}$ & & & & & & \\
\hline \multicolumn{9}{|c|}{$\chi^{2}: 59.3, p=0.356$} \\
\hline
\end{tabular}

*Estado de Puebla.

VP: venas pulmonares; GA: grandes arterias.

Confidencialidad de los datos. Los autores declaran que han seguido los protocolos de su centro de trabajo sobre la publicación de datos de pacientes.

Derecho a la privacidad y consentimiento informado. Los autores han obtenido el consentimiento informado de los pacientes y/o sujetos referidos en el artículo.

\section{Bibliografía}

1. Oster M. Newborn screening for critical congenital heart disease using pulse oximetry [Internet]. UpToDate; 2017 [consultado: 17 septiembre 2020]. Disponible en: https://www.uptodate.com/contents/newborn-screening-for--congenital-heart-disease-using-pulse-oximetry

2. Medrano C, Guía J, Rueda F, Moruno A. Actualización en cardiología pediátrica y cardiopatías congénitas. Rev Esp Cardiol, Supl. [Internet]. 2009 [consultado: 17 septiembre 2020];9(1):39-52. Disponible en: https:// www.sciencedirect.com/science/article/abs/pii/S1131358709717716

3. Perich R, Subirana M, Malo P. Temas de actualidad en cardiología pediátrica y cardiopatías congénitas. Rev Esp Cardiol [Internet]. 2006 [consultado: 17 septiembre 2020];59(Supl.1):87-98. Disponible en: https:// www.sciencedirect.com/science/article/abs/pii/S0300893206748126

4. Valentín A. Cardiopatías congénitas en edad pediátrica, aspectos clínicos y epidemiológicos. Rev Med Electron [Internet]. 2018 [consultado: 17 septiembre 2020];40(4):1083-99. Disponible en: http://scielo.sld.cu/ scielo.php?pid $=$ S1684-18242018000400015\&script $=$ sci_arttext\&tlng $=$ en

5. van der L, Konings $E$, Slager $M$, Witsenburg $M$, Helbing WA, Takkenberg JJ, et al. Birth prevalence of congenital heart disease worldwide. A systematic review and meta-analysis. J Am Coll Cardiol. 2011;58:2241-7.
6. Uribe Rivera AK, Díaz-Vélez C, Cerrón-Rivera C. Características epidemiológicas y clínicas de las cardiopatías congénitas en menores de 5 años del Hospital Almanzor Aguinaga Asenjo. Enero-diciembre 2012. Horiz Med. 2015;15(1):49-56.

7. Fonseca L, Bobabilla J. Abordaje del niño con sospecha de cardiopatía congénita. Rev Mex Pediatr. 2015;82(3):104-13.

8. Mendieta-Alcára G, Santiago-Alcántara E, Méndieta-Zerón H. Incidencia de las cardiopatías congénitas y los factores asociados a la letalidad en niños nacidos en dos hospitales del Estado de México. Gac Med Mex. 2013;149:617-23.

9. Vecoli C, Pulignani S, Foffa I, Andreassi MG. Congenital heart disease: The crossroads of genetics, epigenetics and environment. Curr Genomics. 2014;15:390-9.

10. Blanco Pereira ME, Almeida Campos S, Russinyoll Fonte G, Rodríguez de la Torre G, Olivera Muñiz EH, Medina Robainas R. Actualización sobre la cardiogénesis y epidemiología de las cardiopatías congénitas. Rev Med Electron [Internet]. 2009 [consultado: 17 de septiembre de 2020];31(3). Disponible en URL: http://www.revmatanzas.sld.cu/revista\%20medica/año\%202009/vol3\%202009/tema11.html

11. Valentín Rodríguez A. Cardiopatías congénitas en edad pediátrica, aspectos clínicos y epidemiológicos. Rev Med Electron [Internet]. 2018 [consultado: 23 de septiembre de 2020];40(4). Disponible en: http://www. revmedicaelectronica.sld.cu/index.php/rme/article/view/2479/3971

12. Qu Y, Liu X, Zhuang J, Chen G, Mai J, Guo X, et al. Incidence of congenital heart disease: The 9-year experience of the Guangdong Registry of Congenital Heart Disease. China. PLoS One. 2016;11:1-12.

13. Solano-Fiesco L, Aparicio-Osorio M, Romero-Ramírez J. Prevalencia e incidencia de cardiopatías congénitas en el Servicio de Cardiología Pediátrica del Hospital Central Militar; enero 2006-enero 2010. Rev Sanid Milit Mex. 2015;69:171-8.

14. Kumar $P$. Universal pulse oximetry screening for early detection of critical congenital heart disease. Clin Med Insights Pediatr. 2016;10:35-41.

15. Vázquez $\mathrm{C}$, Alva $\mathrm{C}$, Yañez $\mathrm{L}$, Márquez $\mathrm{H}$. Las cardiopatías congénitas en el 2018. Gac Med Mex. 2018;154:698-711.

16. Varela J, Contreras E, Calderón J, Ramírez S, Cervantes J, Patiño E, et al. Epidemiología de pacientes con cardiopatías congénitas sometidos a cirugía en un hospital privado de tercer nivel en México. Rev Invest Med Sur Mex. 2015;22(4):182-8. 